\title{
Use of near-infrared spectroscopy to probe occlusion severity in patients diagnosed with carotid atherosclerotic disease
}

\section{Authors:}

\author{
Edwin J. Forero ${ }^{1, *}$ \\ Sergio L. Novi ${ }^{1, *}$ \\ Wagner M. Avelar ${ }^{2}$ \\ Carlos A. Anjos ${ }^{1}$ \\ Julien G. Menko ${ }^{1,3}$ \\ Rodrigo M. Forti ${ }^{1}$ \\ Vinicius R. Oliveira ${ }^{1}$ \\ Fernando Cendes ${ }^{2,4}$ \\ Roberto J. M. Covolan ${ }^{1,4}$ \\ Rickson C. Mesquita ${ }^{1,4}$ \\ * The authors contributed equally to this \\ work
}

\section{Affiliations:}

Institute of Physics, University of Campinas, Campinas, Brazil

2 Department of Neurology, University of Campinas, Campinas, Brazil

3 Albert Einstein College of Medicine, Bronx, NY, USA

4 Brazilian Institute of Neuroscience and Neurotechnology, Campinas, Brazil

\section{Corresponding Author:}

Rickson C. Mesquita, Ph.D.

777 Sergio Buarque de Holanda St., DRCC/IFGW

Campinas, SP, Brazil 13083-859

Telephone: +55 (19) 3521-0137

E-mail: rickson@ifi.unicamp.br

\section{Abstract:}

Atherosclerotic disease has been associated with increased risk of severe neurovascular consequences including transient ischemic attacks, ischemic strokes, and even mortality. Following the onset of carotid stenosis, the brain undergoes different compensatory mechanisms to provide adequate perfusion in order to maintain brain metabolism. In this study, we employed near-infrared spectroscopy (NIRS) to better comprehend how carotid stenosis affects cerebral hemodynamics, both at rest and during activity. Eighteen patients diagnosed with atherosclerotic disease were recruited for a protocol consisting of resting state and vasoreactivity testing performed with breath holding. Although the breath holding challenge induced global vasodilation in all patients, the extent of dilation varied according to the level of stenosis. Patients diagnosed with carotid stenosis have impaired hemodynamic response, with a median $77 \%$ vasoreactivity in the hemisphere ipsilateral to the stenotic vessel when compared to the healthy hemisphere. Hemodynamics of these patients differed at rest, with 33\% fewer network links in the hemisphere ipsilateral to the stenosis than the healthy hemisphere. On the other hand, hemodynamic patterns were more heterogeneous with patients diagnosed with a carotid occlusion, which correlates with the opening of collateral circulation. Overall, our results suggest that NIRS can open new directions to the investigation of the effects of cerebrovascular atherosclerotic disease.

Keywords: NIRS, stenosis, breath holding, functional connectivity

Conflicts of Interest: The authors declare no conflict of interest. 
Medical Research Archives, Vol. 5, Issue 6, June 2017

Use of near-infrared spectroscopy to probe occlusion severity in patients diagnosed with carotid atherosclerotic disease

\section{Introduction}

Atherosclerotic disease at the carotid bifurcation has been associated with increased risk of severe neurovascular consequences including transient ischemic attacks (Duncan et al., 1976; Fisher, 1962), ischemic strokes (Hollander et al., 2002), and even mortality (Störk et al., 2004). These pathologies stem not only from the stenosis of the carotid artery by the atherosclerotic plaque resulting in diminished cerebral blood flow through the narrowed lumen, but also via rupture and subsequent thrombosis of the atheroma, or embolic events (Babikian et al., 1994; Carr et al., 1996; Delcker et al., 1997; Philip et al., 1984). Studies have shown that the incidence and severity of carotid stenosis increase with age (de Weerd et al., 2010), and can be detected in as many as $75 \%$ of men (O'Leary et al., 1992). While the prevalence of carotid atherosclerosis and its sequellae have been well documented, not much is known about the brain's compensatory mechanisms.

Carotid artery disease is clinically assessed with carotid duplex ultrasound (CDUS), CT angiography (CTA), MR angiography (MRA), and/or conventional cerebral angiography (CCA). CCA is not common anymore since it confers a high risk of morbidity and mortality due to its invasive nature (Hankey et al., 1990). CTA and MRA are both noninvasive imaging techniques that are commonly used in the evaluation of carotid atherosclerotic disease. While these are both highly accurate techniques for assessing high-grade carotid stenosis (Debrey et al., 2008; Wardlaw, 2006), there are numerous limitations including lack of portability, the inability to make continuous measurements, and the radiation risk from CTA. Additionally, these techniques often require use of IV contrast agents that are contraindicated for patients with renal disease. CDUS has the advantage of being non-invasive, inexpensive, and it has been shown to be both sensitive and specific for detecting stenosis (Carroll, 1991; Sabeti et al., 2004; Zwiebel, 1992). Transcranial Doppler ultrasound (TCD) is an adjunct to CDUS that examines the intracranial hemodynamics, and it is useful for assessing the development of collaterals in the circle of Willis (Wilterdink et al., 1997). Despite these advantages, ultrasound technologies are operator dependent and less precise in measuring lower degrees of stenosis (Carroll, 1991). Additionally, they can be limited by calcification in the carotid plaques, or the patient's body habitus.

Using these imaging techniques, several different models have been proposed to calculate the degree of stenosis (North American Symptomatic Carotid Endarterectomy Trial (NASCET) Steering Committee, 1991; Rothwell et al., 1994; Trial, 1991). Some of these techniques have also been useful in assessing impaired vasomotor reactivity, which is an important adverse prognostic factor in patients with carotid stenosis. Among several different protocols to induce vasoreactivity, the simplest hemodynamic challenge (which is commonly used in the clinic) is the breath holding task. For example, one can implement TCD to estimate the breathholding index (BHI) to evaluate cerebral vasoreactivity by measuring changes in the cerebral blood flow velocity of proximal arteries of the circle of Willis (Vasdekis et al., 2012). Although TCD-based BHI has been shown to correlate with the degree of stenosis, changes in the microvasculature should reflect vasomotor reactivity more directly to the tissue level. In addition, not all patients have temporal acoustic windows available for TCD (Larsen et al., 1994; Tsivgoulis et al., 2007)

Near-infrared spectroscopy (NIRS) has been highlighted as a non-invasive brain 
monitoring technique (Ferrari and Quaresima, 2012; Mesquita and Yodh, 2011; Scholkmann et al., 2014; Yodh and Boas, 2003). NIRS uses the fact that near-infrared light $(\sim 700-900 \mathrm{~nm})$ is weakly absorbed by biological tissue and therefore can penetrate several centimeters deep. In the nearinfrared region of the electromagnetic spectrum the main absorber in tissue is hemoglobin. Concurrently, tissue strongly scatters near-infrared light, which makes possible to detect (scattered) light from the same plane of incidence (Jobsis, 1977). In functional NIRS experiments, which aim to probe brain function, near-infrared light emitted by a light source from the scalp penetrates deep enough to probe the most external surface of the cortex, and part of the scattered light is detected on the scalp few centimeters away from the source. Over time, the detected light carries information about the changes in tissue absorption due to changes in hemoglobin concentration. Therefore, NIRS can recover information about cortical hemodynamics at the microvasculature, which provides a clearer measure of brain function. NIRS' portability, high temporal resolution, low cost and ease of use make it a suitable tool to be used on several environments, ranging from research laboratory to the patient's bedside, as well as in remote areas (Favilla et al., 2014; Mesquita et al., 2013, 2012, 2009; Sanborn et al., 2015). However, NIRS is limited to probe hemodynamic activity to more superficial regions, since scattered light from deeper structures are absorbed before reaching the detector.

We hypothesize that NIRS can be employed to further understand how different levels of carotid stenosis affect brain function on a microvascular level. In this pilot study, we measured the NIRS response of 18 patients diagnosed with carotid stenosis under two different conditions: during a vasoreactivity (breath

Copyright 2017 KEI Journals. All Rights Reserved holding) task and during the resting state. The vasoreactivity task showed significant differences in the microvascular hemodynamic response patterns due to stenosis, including the inversion of collateral vessels in the hemispheres with decreased blood supply. Similarly, functional connectivity analysis during the resting state indicated abnormal connectivity patterns of patients compared to healthy subjects. In particular, we found a high correlation in the spontaneous hemodynamic fluctuations within the hemisphere contralateral to the stenosis/occlusion along with a lack of interhemispheric connections typically seen in healthy subjects. Taken together, our results suggest that NIRS can open novel options to further understand the effects of stenosis in the brain of patients.

\section{Research Methods}

\subsection{Patients}

This study was conducted in accordance with institutional policies and it was approved by the University of Campinas Institutional Review Board. The experimental protocol is in agreement with the principles from the Helsinki II convention from Aug 20, 1947.

Eighteen patients (10 male) with clinically diagnosed carotid stenosis were recruited to this pilot study. The diagnostics was initially made with carotid ultrasound and then confirmed with CTA of cervical vessels or digital arteriography. All recruited patients underwent 3T MRA to exclude silent stroke. Medical history and neurological examination were performed on all patients in order to exclude a previous history of stroke or transient ischemic accident.

Table 1 shows the demographics of all the patients recruited in this study. The average age of the cohort was $67 \pm 7$ years 
old, ranging from 51 to 75 years old. For our research purposes, the degree of stenosis was classified as normal (i.e., no stenosis), altered (> 50\% stenosis) or occluded (when there was no detectable patent lumen at gray-scale ultrasound). MRA and CTA were also used to analyze collateral circulation at the left and right posterior communicant, and the anterior communicating artery. The breath-holding index (BHI) was assessed for all recruited patients prior to our experimental protocol with TCD (Sonara, Natus Medical, Inc., Pleasanton, CA, USA). The BHI for each patient is also available in Table 1.

Table 1. Detailed characteristics for all patients recruited in this study. The group was classified according to the hemispherical diagnosis (see Section 2.5 for details) as Normal-Altered (NA), Normal-Occluded (NO) or Altered-Occluded (AO). The breath holding index (BHI) was obtained from TCD, and the number of collateral vessels (NCV) opened was obtained from MRA and CTA. (Note: F: Female, M: Male, C: Caucasian, AA: African American.)

\begin{tabular}{ccccccc}
\hline Patient & Age (years) & Gender & Race & Group & NCV & BHI \\
\hline 1 & 71 & F & C & NA & 0 & 1.2 \\
2 & 75 & M & AA & NA & 0 & 1.1 \\
3 & 56 & M & C & AO & 3 & 0.2 \\
4 & 63 & M & C & NO & 2 & 0.5 \\
5 & 68 & M & C & AO & 2 & 0.4 \\
6 & 72 & M & C & NA & 3 & 0.3 \\
7 & 73 & M & C & NA & 0 & 0.3 \\
8 & 60 & F & C & AO & 2 & 0.8 \\
9 & 62 & F & C & NA & 1 & 0.6 \\
10 & 64 & F & AA & NA & 3 & 0 \\
11 & 70 & M & C & NA & 1 & 0.1 \\
12 & 71 & M & C & NA & 0 & 0.9 \\
13 & 51 & F & C & NO & 2 & 0.8 \\
14 & 75 & F & C & NA & 1 & 0.7 \\
15 & 67 & F & C & NO & 2 & 0.6 \\
16 & 59 & M & C & NO & 3 & 0.3 \\
17 & 72 & M & C & NA & 0 & 0.2 \\
18 & 74 & F & C & AO & 2 & 0.7 \\
\hline
\end{tabular}

\subsection{NIRS Experimental Protocol}

The experimental protocol consisted of one single session, in which patients were instructed to sit down in a comfortable chair and relax. All NIRS optodes were positioned on the patient's head. During the experiment, all patients performed two independent tasks. In the first part of the session, patients were required to close their eyes and to not focus on any specific task to reproduce the resting state. For each subject, a 300-second baseline run was performed once. The second part of the protocol was similar to the clinical vasoreactivity test performed with TCD. Briefly, patients were asked to hold their breath for target duration of 30 seconds followed by a rest period of 240 seconds. This procedure was repeated twice for a total measurement of approximately 600 seconds. The whole protocol lasted approximately 1 hour. 
All NIRS data were acquired with a continuous-wave NIRS system (CW6, TechEn Inc., Milford, MA, USA). The temporal resolution of the system was 25 Hz. The NIRS optical probe was designed with 8 laser sources at 2 different wavelengths $(690$ and $830 \mathrm{~nm}$, emitting $10 \mathrm{~mW}$ each) and 16 detectors placed on the scalp to cover most of the frontal and parietal lobes. This geometry configuration allowed 28 different source-detector pairs (channels) with $3 \mathrm{~cm}$ separation between sources and detectors. Figure 1-A shows the regions that the probe was sensitive to, covering hemodynamic changes from the middle frontal, superior frontal, precentral and postcentral gyri.

\subsection{Functional Analysis}

Functional analysis followed established protocols in the NIRS community (Huppert et al., 2009). First, motion artifacts were automatically removed by employing a wavelet filter (Brigadoi et al., 2014). Channels with low signal-to-noise ratio (i.e., SNR < 8) were also discarded at the pre-processing step. The remaining channels were band pass filtered $(0.005-0.5$ $\mathrm{Hz})$ and converted to oxy-( $\mathrm{HbO})$ and deoxy(HbR) hemoglobin concentration changes by applying the modified Beer-Lambert law with a different pathlength factor of 6 for both wavelengths. Total hemoglobin concentration (HbT) was obtained by summing $\mathrm{HbO}$ and $\mathrm{HbR}$. The hemodynamic response due to breath holding was obtained for each channel by averaging the trials, starting 2 seconds prior to the beginning of the task and ending 60 seconds after the completion of the task. Since breath holding is a global task (as it induces vasodilation across all regions of the brain), signals from all channels were averaged in order to estimate a global hemodynamic vasoreactivity response for each patient. Figure 1-B illustrates the analysis procedure.

\section{Analysis \\ 2.4. Resting-State Connectivity}

For the resting state data, regions around motion artifacts (identified as sudden changes in intensity level) were discarded. Similarly to the functional analysis, channels with SNR $<8$ were also not considered for analysis. The remaining channels were bandpass filtered between 0.009 and $0.08 \mathrm{~Hz}$ for analysis of low frequency hemodynamic oscillations (Greicius et al., 2009; Lu et al., 2010; Mesquita et al., 2010; Novi et al., 2016; Sasai et al., 2012), and hemoglobin concentration changes were estimated as in the functional analysis. Last, we decomposed the signal using a Principal Component Analysis (PCA) algorithm, and filtered out the first principal component from the original signal, which has been shown to be strongly correlated with the global average signal (Carbonell et al., 2011; Novi et al., 2016).

The NIRS-based network was constructed from the correlation matrix between the time-courses of each of the hemoglobin concentrations (Novi et al., 2016, Figure 1C). In the connectivity network, the nodes represent the channels and the links represent the similarity (as measured by the Pearson correlation coefficient) between two given nodes. For each network, we arbitrarily fixed the average number of links to correspond to $20 \%$ of the maximum number of links. The average degree was calculated for each network and employed as a measure to how connected the different regions are. In order to derive common patterns across the whole population, we first separated the hemispheres occluded/ipsilateral to the stenosis from the normal hemispheres, and then calculated a frequency network by computing the frequency that each link appears in the network across all patients (Novi et al., 2016). 
A)
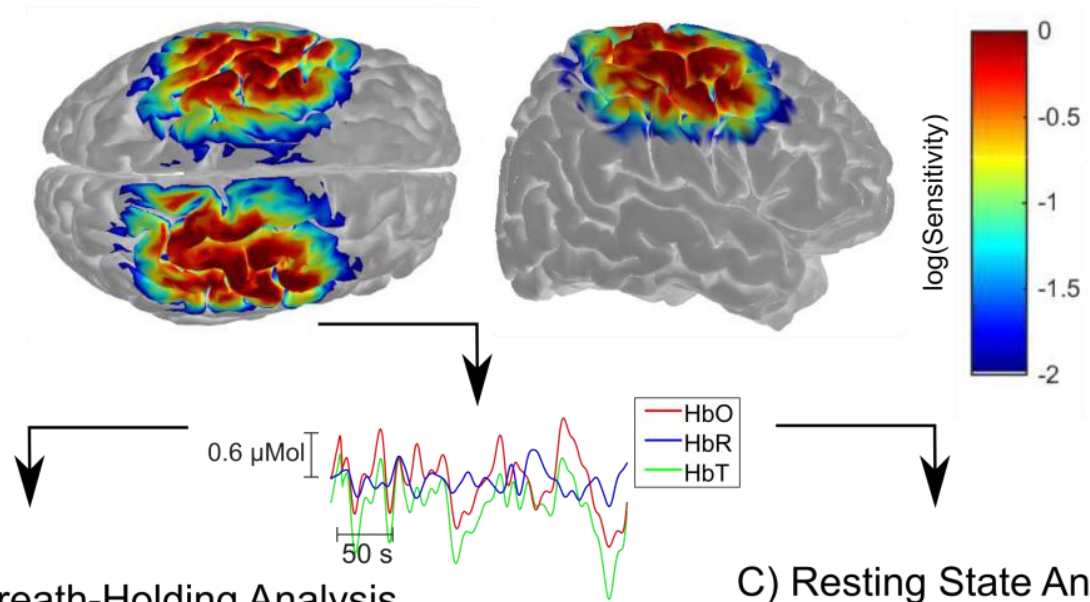

B) Breath-Holding Analysis

C) Resting State Analysis

Experimental Design

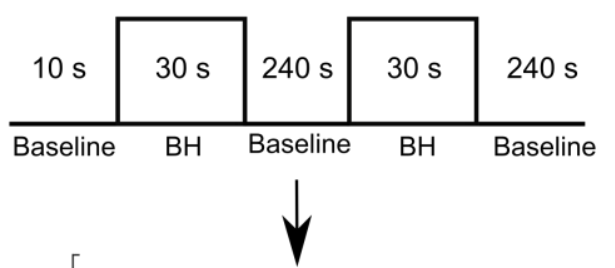

Correlation Matrix
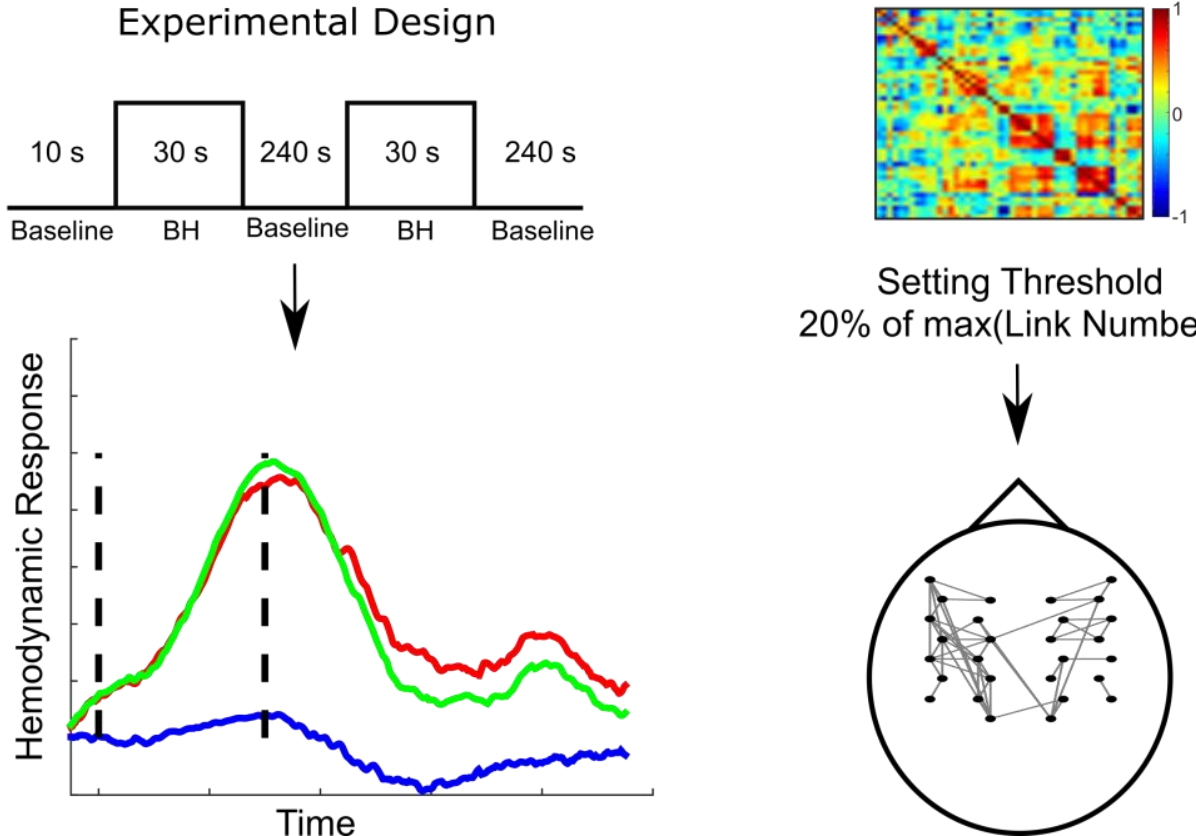

Setting Threshold $20 \%$ of max(Link Number)

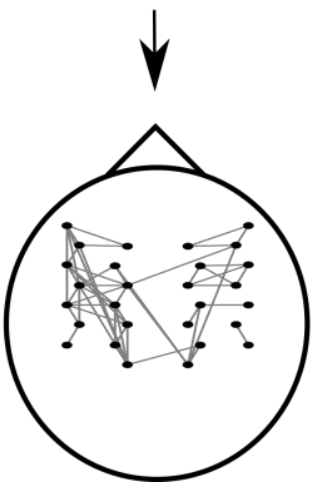

Figure 1. Details of the experimental protocol and analysis methods. (A) Monte Carlo simulation of the photon fluence sensitivity for the probe configuration employed in this study. Simulation was performed with $10^{8}$ photons (Aasted et al., 2015). Color bar indicates the sensitivity of our probe in a logarithmic scale (red represents regions with highest sensitivity). The simulation suggests that measurements are more sensitive to frontal, superior frontal, precentral and postcentral gyri. Oxy- $(\mathrm{HbO})$, deoxy- $(\mathrm{HbR})$ and total-hemoglobin changes (HbT) were estimated from the regions measured. (B) Schematics of the functional protocol, which consisted of two breath holding trials. The hemodynamic response was obtained by averaging the NIRS time-series around the task periods for each channel. (C) For the restingstate connectivity analysis, we computed correlation matrices independently for $\mathrm{HbO}, \mathrm{HbR}$ and $\mathrm{HbT}$ by calculating the Pearson correlation coefficient across all channels, and then we kept the links between the highest correlation coefficients until we reach the threshold of $20 \%$ of the maximum number of possible links. The final graph was back projected onto 2D topographic maps of the optical geometry for visual analysis. 


\subsection{Statistical Analysis}

Patients were separated in three different groups according to their diagnosis: 1) the normal-altered (NA) group, which presented one normal hemisphere and another one altered (i.e., > 50\% stenosis); 2) the normal-occluded (NO) group, with one normal and one occluded hemispheres, and; 3 ) the altered-occluded (AO) group, in which one hemisphere was classified as altered and another one with occlusion. In total, we had 10 patients in the NA group, 4 patients in the NO group, and 4 patients in the AO group. Data from the groups were summarized using the median and interquartile range (IQR). Since the main goal of this pilot study was to better understand hemodynamic activity of different patients, we did not aim to address group comparisons. Instead, we analyzed individual patterns and how common such patterns are in patients with the same diagnostics.
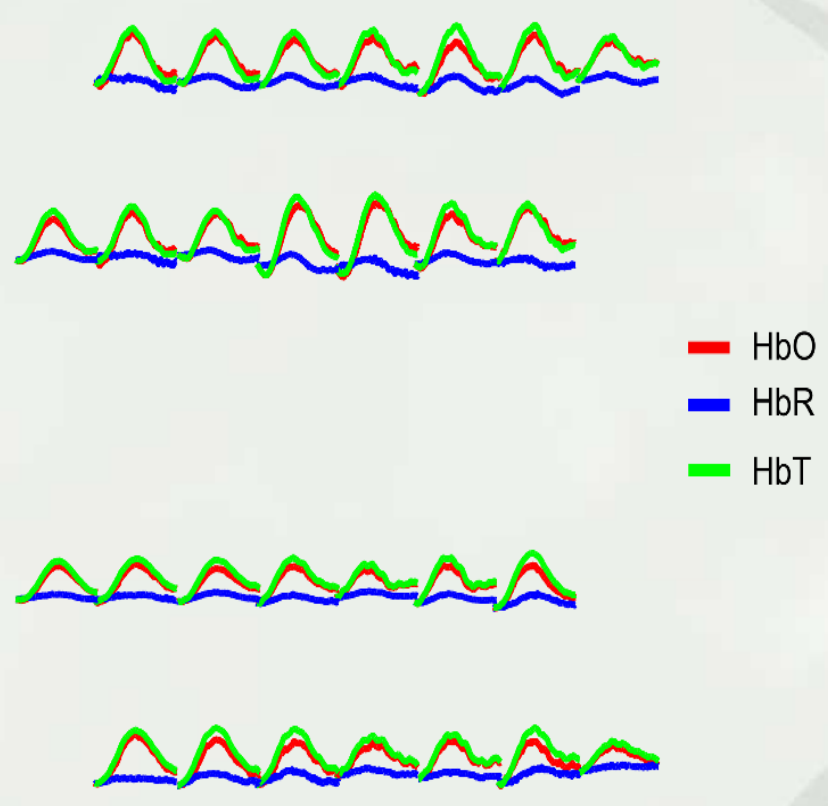

Figure 2. Hemodynamic response due to the vasoreactivity challenge for a representative patient. The oxy- $(\mathrm{HbO})$, deoxy- $(\mathrm{HbR})$ and total-hemoglobin $(\mathrm{HbT})$ concentration changes were observed in all regions measured, and it was characterized by a significant increase in $\mathrm{HbO}$ and $\mathrm{HbT}$, with an increase followed by a decrease in HbR. The hemodynamic changes return to baseline few seconds after the task is over. 
Medical Research Archives, Vol. 5, Issue 6, June 2017

Use of near-infrared spectroscopy to probe occlusion severity in patients diagnosed with carotid atherosclerotic disease

\section{(A) Normal-Altered}
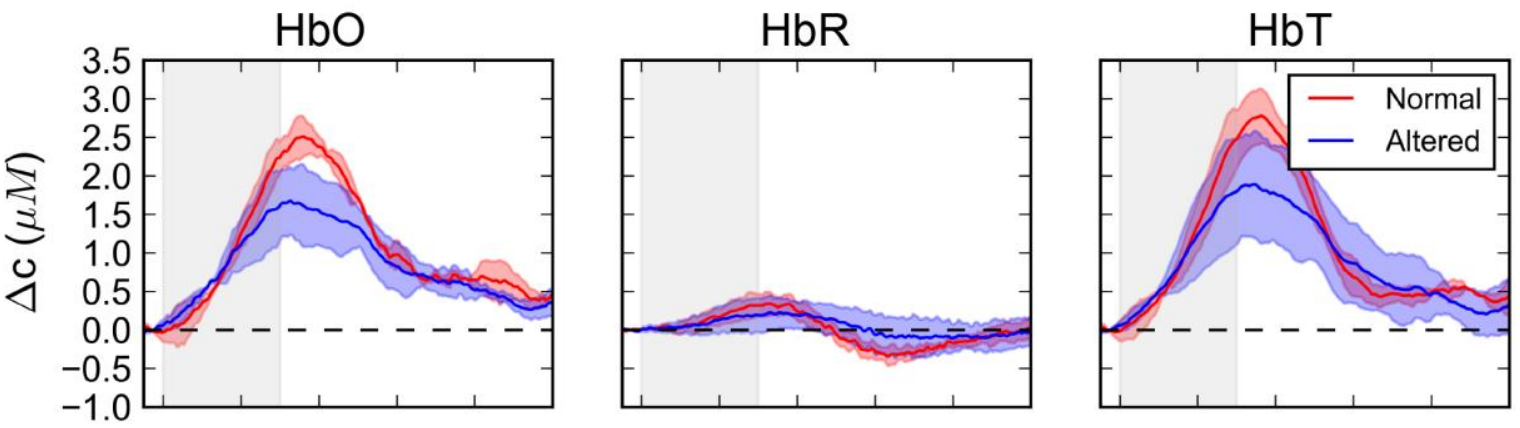

(B) Normal-Occluded
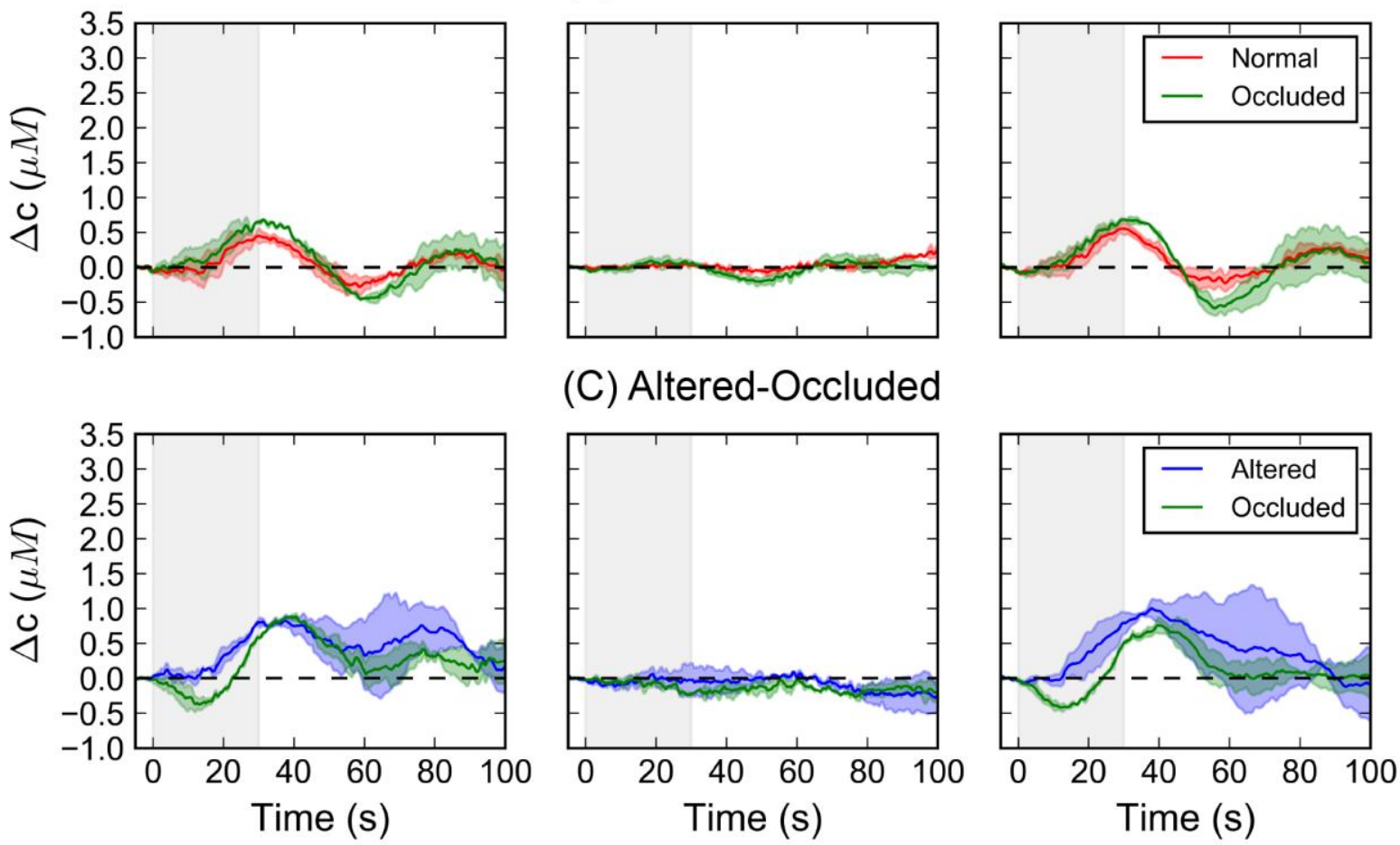

Figure 3. Average hemodynamic concentration changes $(\boldsymbol{\Delta c})$ due to breath holding for oxy- (HbO, first column), deoxy- (HbR, second column) and total-hemoglobin (HbT, third column) concentrations, separated by hemisphere, for one representative patient of each group: (A) normal-altered (NA), (B) normal-occluded (NO) and $(\mathrm{C})$ altered-occluded (AO). In the plots, the gray region represents the breath holding period. The solid curves are the mean response across all channels from the hemisphere, and the shadows represent the standard deviation across all trials and channels.

\section{Results}

\subsection{Breath holding induces a global hemodynamic response in all patients}

Figure 2 shows the average $\mathrm{HbO}, \mathrm{HbR}$ and $\mathrm{HbT}$ responses during the breathholding period for each source-detector position for a representative patient. As expected, we observed significant increases of $\mathrm{HbO}$ and $\mathrm{HbT}$ in all channels during the first 10 seconds of the task, which can be associated to the vasodilation as a consequence of the increase in partial pressure of $\mathrm{CO}_{2}$. The increase in $\mathrm{HbO}$ and HbT synchronously reached their maximum 
value near the end of the task. The HbR response was more heterogeneous, showing a slight increase at the beginning of the task followed by a decrease near the end of the task in most of the cases. In all cases, the hemoglobin concentrations decreased to the baseline value from 15 to 20 seconds after the end of the task. This global behavior, similar to what previous studies have seen for healthy subjects, was observed in all patients, which suggests that the stenosis degree does not affect the characteristic hemodynamic response during a vasoreactivity task.

\subsection{Hemodynamic changes induced by breath holding vary with stenosis degree}

In order to compare the behavior of the hemodynamic response in each hemisphere, we calculated the median response over all channels located at the same hemisphere of each subject during the breath-holding task.
Figure 3 shows the characteristic hemoglobin concentration changes for each hemisphere for a representative patient of each group. Although the hemodynamic response has similar dynamics across both hemispheres in all groups, the amplitude of the hemispheric vasodilation can be quite different. For all the patients in the NA group, the BH-induced hemodynamic change in the hemisphere ipsilateral to the stenosis was significantly smaller than the hemodynamic change in the contralateral hemisphere. The median (IQR) change in the stenosis/normal hemispherical $\mathrm{HbO}$ ratio was $0.77(0.58-0.89)$, i.e., the $\mathrm{HbO}$ increase in the hemisphere with stenosis was $77 \%$ of the increase in the normal hemisphere. Similarly, the HbT increase in the hemisphere ipsilateral to the stenosis was smaller than in the contralateral hemisphere (median $=0.74$, IQR: $0.56-0.90)$. Table 2 summarizes all hemispherical changes by group.

Table 2. Median (IQR) hemispherical ratio of the changes in oxy- (HbO), deoxy- (HbR) and total-hemoglobin $(\mathrm{HbT})$ across all patients for each group. The ratio was calculated by dividing the maximum change of $\mathrm{Hb}$ in each hemisphere across all channels. (NA: normal-altered group; NO: normal-occluded group; AO: altered-occluded group.)

\begin{tabular}{cccc}
\hline \multirow{2}{*}{ Hemodynamic contrast } & \multicolumn{3}{c}{ Patient Groups } \\
\cline { 2 - 4 } & NA & NO & AO \\
\hline HbO & $0.77(0.58-0.89)$ & $1.32(1.31-1.33)$ & $0.89(0.81-0.97)$ \\
$\mathrm{HbR}$ & $0.87(0.78-1.59)$ & $0.74(0.73-0.75)$ & $1.15(1.07-1.22)$ \\
$\mathrm{HbT}$ & $0.74(0.56-0.90)$ & $1.16(1.13-1.19)$ & $0.95(0.76-1.15)$ \\
\hline
\end{tabular}

Interestingly, the hemodynamic response in the occluded hemisphere was not smaller than the hemodynamic response in the normal hemisphere for all patients in the NO group. Patients in the AO group presented a slightly smaller response in the occluded hemisphere when compared to the stenosis hemisphere, but very close to 1 . Collateral circulation can explain the similarity of the hemodynamic responses between the two hemispheres in the occluded patients. In our cohort, all patients with an occluded hemisphere had at least 2 communicating arteries opened, which did not happen to the NA group (Table 1).

\subsection{Carotid stenosis affects hemodynamic activity at rest}

Last, we investigated how spontaneous 
hemodynamic activity is affected in patients diagnosed with carotid stenosis. Figure 4 shows the NIRS-based networks for representative patients in the NA, NO and AO groups. In all cases, it is possible to note that the number of links is higher in the normal hemisphere (for the NA and NO groups), suggesting that the disease disturbs the coherence of the hemodynamic fluctuations even at rest.

In order to quantify the average number of links, we calculated the ratio of the number of links between the hemispheres. Table 3 summarizes the results for all contrasts as function of the group. For the $\mathrm{HbO}$ networks in the NA group, the median (IQR) number of links in the normal hemisphere was 1.5 (1.2 - 1.8) times the number of links in the altered hemisphere. Similarly, this ratio was $1.2(1.1-1.7)$ for the HbT networks. We did not observe any pattern in the HbR maps; the median ratio for the NA group was $1.0(0.7-1.5)$. Patients with an occluded hemisphere did not exhibit any standard trend, with ratios oscillating around 1.

Table 3. Median (IQR) hemispherical ratio of the number of links across all patients. The number of links was calculated across all nodes in the hemisphere based on the functional connectivity during the resting state, for each NIRS-based contrast: oxy- (HbO), deoxy- (HbR) and totalhemoglobin (HbT). (NA: normal-altered group; NO: normal-occluded group; AO: alteredoccluded group.)

\begin{tabular}{cccc}
\hline \multirow{2}{*}{ Hemodynamic contrast } & \multicolumn{3}{l}{ Patient Groups } \\
\cline { 2 - 4 } & NA & NO & AO \\
\hline $\mathrm{HbO}$ & $1.5(1.2-1.8)$ & $1.2(1.0-1.5)$ & $1.0(0.9-1.1)$ \\
$\mathrm{HbR}$ & $1.0(0.7-1.5)$ & $0.9(0.7-1.3)$ & $0.5(0.3-0.8)$ \\
$\mathrm{HbT}$ & $1.2(1.1-1.7)$ & $1.2(1.0-1.4)$ & $1.3(1.2-1.4)$ \\
\hline
\end{tabular}

\subsection{Spontaneous hemodynamic fluctuations are more heterogeneous in patients with carotid stenosis}

Next, we were interested in finding the common links (brain connections) across the whole population. Figure $5 \mathrm{~A}$ shows the resultant networks with the most frequent links across all patients for each NIRS contrast. It is possible to note that the common links in all contrasts are predominantly short-range links (i.e., links between close regions in the brain). In addition, the hemisphere ipsilateral to the stenosis/occlusion has considerably fewer links than the contralateral hemisphere for all NIRS contrasts.

At the same time, it is striking that the $\mathrm{HbO}, \mathrm{HbR}$ and $\mathrm{HbT}$ resultant networks from the patients lack robust inter-hemispheric connections. In fact, there are only 2 interhemispheric connections with frequency equal or higher than $50 \%$ in the $\mathrm{HbO}$ network and none in the others. Since individual maps of patients do show interhemispheric connections (Figure 4), the lack of inter-hemispheric and long-range links in the resultant maps indicates that these connections are very specific to each patient. This may be related to how the brain compensates for the lack of blood availability, and it is different from what previous studies have found in healthy patients (Novi et al., 2016). By comparison, Figure 5B shows resultant networks obtained for the same number of subjects within a healthy population cohort (collected separately). 
Medical Research Archives, Vol. 5, Issue 6, June 2017

Use of near-infrared spectroscopy to probe occlusion severity in patients diagnosed with carotid atherosclerotic disease

A)

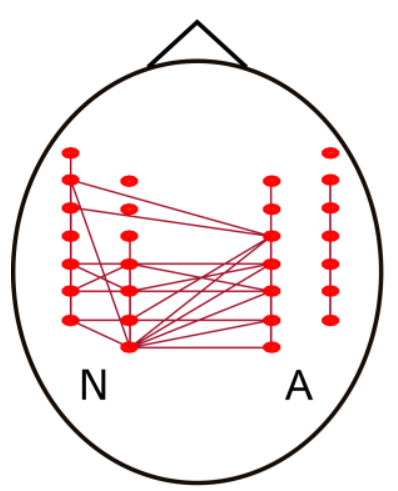

B)

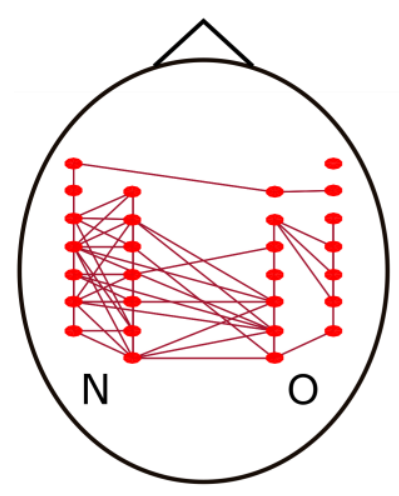

C)

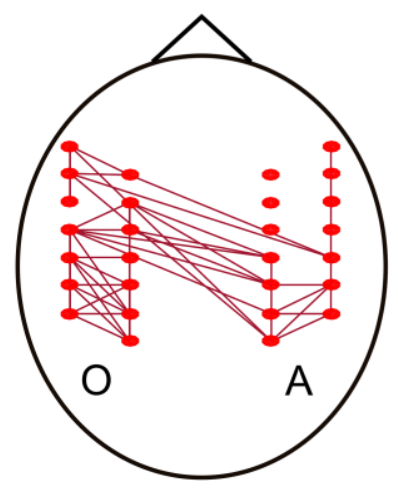

Normal-Altered

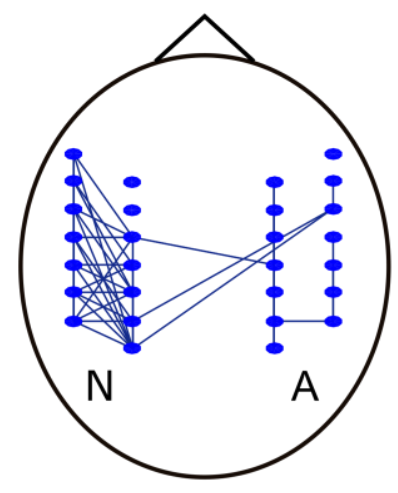

Normal-Occluded
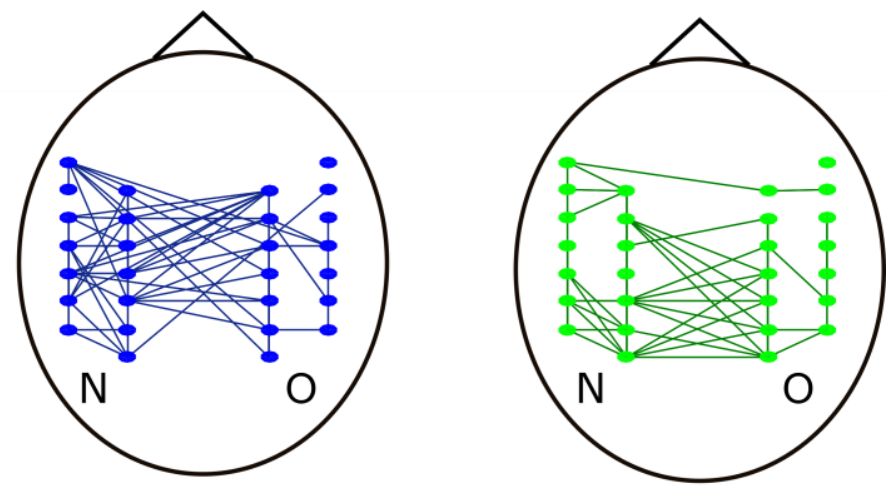

Altered-Occluded
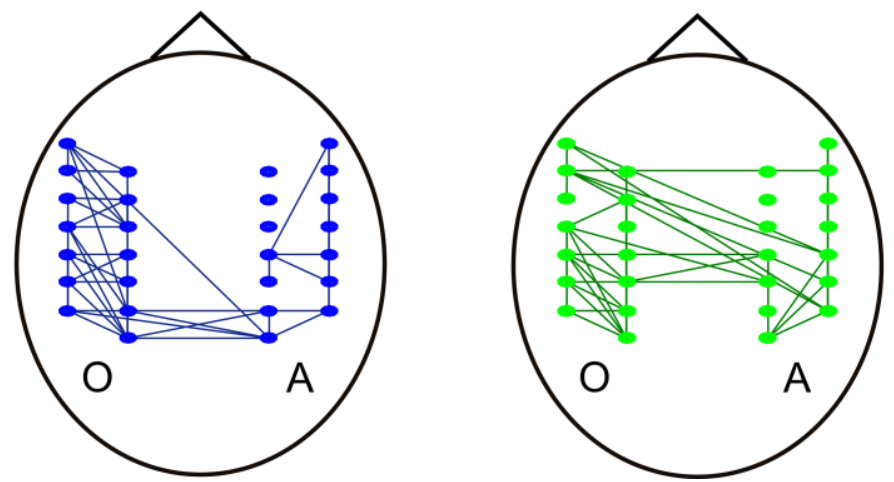

Figure 4: Functional connectivity graphs during the resting state based on oxy- (red, first column), deoxy- (blue, second column) and total-hemoglobin (green, third column) concentration fluctuations for one representative patient of each group: (A) normal-altered (NA), (B) normal-occluded (NO) and (C) altered-occluded (AO). In the plots, the full circles represent every source-detector measurement and the solid lines are the links between different regions measured (i.e., regions with high similarity as measured by the Pearson correlation coefficient). 
A)

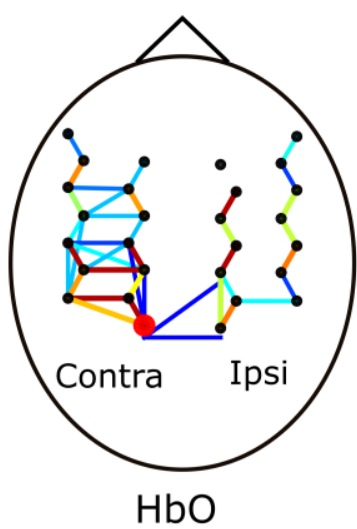

B)

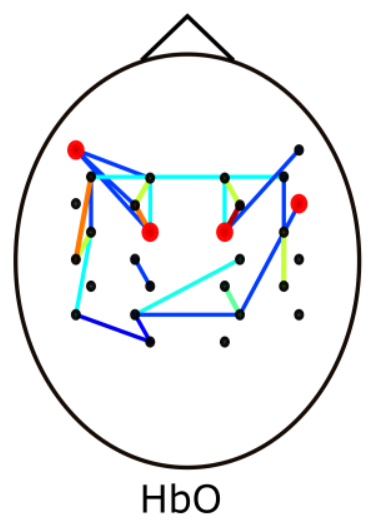

Patients

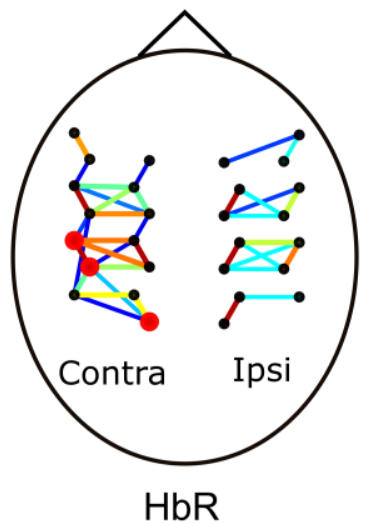

Healthy Group

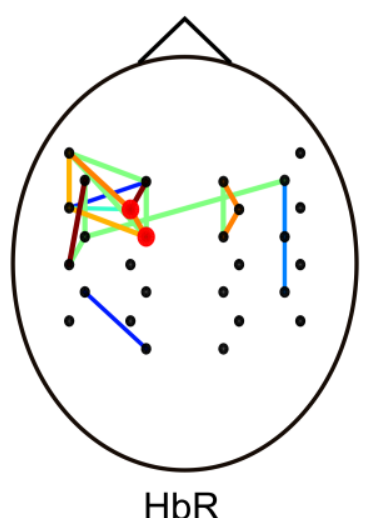

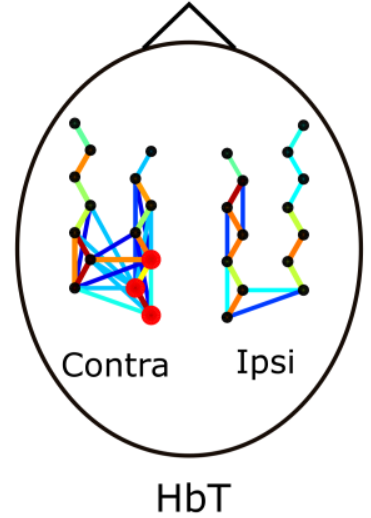

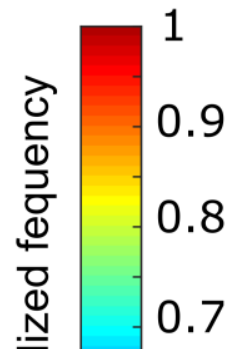

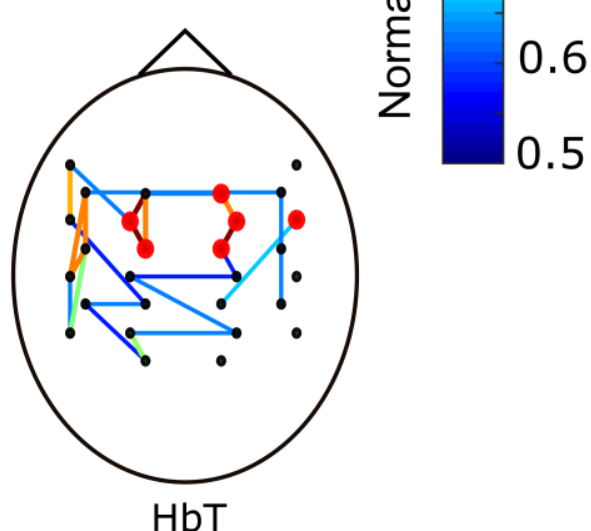

Figure 5. (A) Frequency network for all links across all patients, for each NIRS contrast (HbO: oxyhemoglobin; HbR: deoxy-hemoglobin; HbT: total-hemoglobin). In order to derive the frequency map for all patients, we grouped all hemispheres ipsilateral to the stenosis or occlusion (Ipsi in the figure); normal hemispheres were in the contralateral hemisphere (Contra in the figure). (B) Frequency map for a subset of healthy subjects collected separately for comparison. It is possible to note that healthy subjects present more inter-hemispheric connections in the frequency maps. In the maps, black circles represent the nodes of the graph (NIRS channels), and red dots are the highly connected nodes (hubs). Each link color corresponds to the frequency that the link appeared in each subject group. The frequencies range from 0.5 $(50 \%)$ to $1(100 \%)$.

It is worth mentioning that we arbitrarily established a frequency threshold to quantify the connectivity of each hemisphere. To evaluate the influence of our choice, we varied the threshold from $50 \%$ to $80 \%$ in steps of $5 \%$. Figure 6 shows the ratio of the number of links between the two hemispheres as function of the frequency of the links in the network. It is possible to note that, regardless of the frequency analyzed, the hemisphere contralateral to the stenosis/occlusion has $149 \pm 9 \%$ (mean \pm SD) more links than the hemisphere ipsilateral to the stenosis/occlusion, for all NIRS contrasts. 
Medical Research Archives, Vol. 5, Issue 6, June 2017

Use of near-infrared spectroscopy to probe occlusion severity in patients diagnosed with carotid atherosclerotic disease

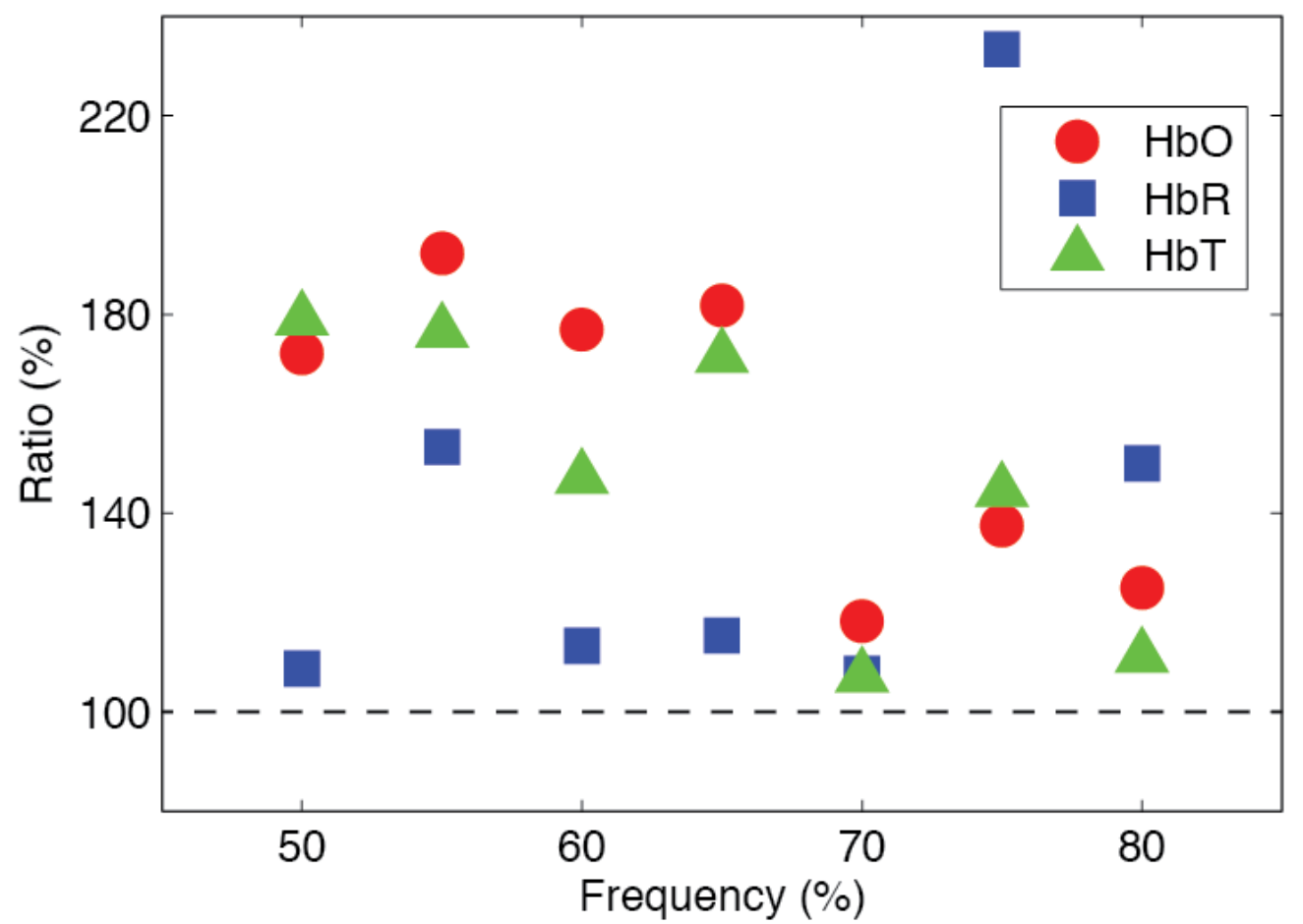

Figure 6. Percent ratio of the number of links between the two hemispheres as function of the frequency threshold for oxy- (HbO), deoxy- (HbR) and total-hemoglobin (HbT) frequency graphs. In all cases studied, the number of links in the hemisphere contralateral to the stenosis/occlusion has more links than in the hemisphere ipsilateral to the stenosis.

Last, in order to further evaluate the importance of each hemisphere in respect to the whole network, we questioned whether there were hubs in the NIRS networks at rest. Hubs are highly connected nodes. To find these important nodes, we computed the weighted degree, $w$, and then we defined as a hub every node that had $\mathrm{w}>90 \%$ of the maximum w of each network (Barrat et al., 2004; Newman, 2004; Novi et al., 2016). Interestingly, all hubs from the patients' networks were located on the hemisphere contralateral to the stenosis/occlusion for $\mathrm{HbO}, \mathrm{HbR}$ and $\mathrm{HbT}$ networks (red nodes in Figure 5). Again, this is a different pattern that one would find in a healthy population, in which hubs are located in both hemispheres (Novi et al., 2016). The predominance of hubs in the hemisphere contralateral to stenosis/occlusion may indicate the importance of this hemisphere to sustain hemodynamics in patients diagnosed with atherosclerotic disease.

\section{Discussion}

In this work, we aimed to investigate whether NIRS could be employed as a complementary tool to better understand the effects of atherosclerotic disease in the brain. In the clinical practice, flow response to vasomotor reactivity is the standard method to infer the effects of carotid stenosis to the brain (Markus and Harrison, 1992; Müller and Schimrigk, 1996; Ratnatunga and Adiseshiah, 1990; Ringelstein et al., 1988; Silvestrini et al., 2009, 1996; Soinne et al., 2003; Vernieri et al., 1999; Widder et al., 1994). Although breath holding is not a 
controlled vasoreactivity task (i.e., it does not induce the same vasodilatory response to all patients), it appears as an affordable and efficient way to induce vasoreactivity in patients and controls. In addition, breath holding has been clinically employed in hospitals, at least in places with limited financial resources. Therefore our research protocol has special relevance to atherosclerotic disease care in low- and middle-income countries.

Previous recent work has shown that NIRS-based vasoreactivity, as measured by a breath-holding index (BHI), correlates with TCD-based vasoreactivity employed in the clinic (Vasdekis et al., 2012). However, the variability in the $\mathrm{BHI}$ as function of patient condition is still subject of debate. Here we chose to address the question of how different patients respond to the breath holding task. Overall, we measured an increase in oxygenation that can be explained by a vasodilatory effect induced by the breath holding (Figure 2). This increase was observed in all patients and in all channels.

One intrinsic limitation of NIRS is its depth penetration. While many studies have shown that NIRS is sensitive to deep (e.g., cerebral) hemodynamics (Franceschini and Boas, 2004), measurements from structures deeper than the most external surface of the cortex would demand source-detector separations greater than $3.0 \mathrm{~cm}$, which degrades signal-to-noise ratio (SNR). Therefore NIRS measurements reflect changes in cortical hemodynamics, only. More importantly, NIRS signals contain extra-cortical contributions from shallower tissues (e.g., scalp) (Minati et al., 2011; Obrig et al., 2000; Oscillators, 2010; Tachtsidis et al., 2008). Superficial layers account for most of the NIRS signal, and removal of such contamination can be difficult, although possible (Gagnon et al., $2014,2012)$. For this reason, we opted not to compare the hemodynamic response due to breath holding between different subjects, since the extra-cortical contribution affects the amplitude changes and it will depend on each subject anatomy. Therefore, by comparing the ratio between different hemispheres for the same subject we avoid errors due to extra-cortical contributions.

Overall, we found that patients with just a single hemispheric stenosis (i.e., patients in the NA group) present a large disparity between the healthy hemisphere and the hemisphere ipsilateral to the stenosis. This hemispherical difference was true in all cases studied in the NA group, both during the vasoreactivity task (Figure 3 and Table 2) and even during the resting state (Figure 4 and Table 3). Although this result is somewhat expected, since the stenosis allows less blood flow into the brain and stresses the vasculature, it is remarkable that NIRS can reliably reflects this condition so clearly in our dataset.

On the other side, the hemodynamic response of patients with at least one occluded hemisphere (i.e., patients in the NO or AO groups) was more heterogeneous. Despite the heterogeneity, one systematic behavior in these patients was the similarity between the two hemispheres. That may be explained by additional blood circulation due to collateral vessels. It is well established in the literature that brain regions affected by stenosis overcome the low metabolic products income with blood coming of collateral vessels (Demchuk et al., 2000; Norrving et al., 2015; Tsivgoulis et al., 2007). Communicating arteries in the circle of Willis play an important role in patients with carotid stenosis or with occluded carotids since collateral flow in these arteries can be reversed to supply additional blood (Zhu et al., 2015). All patients with an occluded carotid have at least two communicating arteries opened (Table 1). Therefore it is reasonable that the 
hemodynamic response between the two hemispheres is similar in these patients, once flow from a healthy region can be reallocated to the occluded region through the collateral circulation. Overall, blood flow across the whole brain will still be decreased, which explains the smaller changes in amplitude in both hemispheres in the NO and AO groups, as compared to the NA group (Figure 3). The heterogeneity is probably related to the number of collateral vessels opened, but this investigation was beyond the scope of this work.

Another novelty of this study is the use of functional connectivity to analyze the interactions among different brain regions during the resting state. Recent studies have showed that several brain diseases, such as Alzheimer, schizophrenia and epilepsy can lead to aberrant connectivity patterns during the resting state (Garrity et al., 2007; Stam et al., 2007; Tian et al., 2016; Zhang et al., 2017), as compared to healthy patterns. Here, we hypothesized that spontaneous hemodynamic fluctuations of the brain at rest could provide biomarkers to stenosis as well.

In fact, we found that NIRS-based functional connectivity is sensitive to different grades of stenosis, even in the resting state. Similar to what we have found for the vasoreactivity task, patients in the NA group have very different behavior between the hemispheres. Specifically, they lack connections in the hemisphere ipsilateral to the stenosis, suggesting either that there are no interactions between different regions of the diseased hemisphere or that the hemodynamic fluctuations are desynchronized (i.e., out of phase). We also observed the absence of connections in the occluded hemisphere in the $\mathrm{NO}$ and $\mathrm{AO}$ groups, although with a higher variability across different patients.

Copyright 2017 KEI Journals. All Rights Reserved
Last, we attempted to analyze robust patterns during the resting state that could be associated with atherosclerotic disease, despite individual variations reported in our work. Since the number of patients in this study was low, we opted to derive a resultant graph based on the frequency of appearance of links rather than taking group averages of properties (Figure 5). This approach allowed us to analyze how similar the graphs are across all patients. In addition, the topological features from the frequency networks can provide insights how atherosclerotic diseases affect spontaneous hemodynamic activity. For example, most of functional connectivity studies with NIRS in healthy subjects show inter-hemispheric connections (Li et al., 2015; Mesquita et al., 2010; Novi et al., 2016; Sakakibara et al., 2016). In this study we were also able to find similarities between hemispheres for all of the patients, which resulted in interhemispheric connections. However, we found inter-hemispheric connections to vary in a great extent across patients, which resulted in a lack of inter-hemispheric connections in the resultant graph based on the frequency of appearance of each link.

Another interesting result from the resultant network is that all hubs were exclusively located in the hemisphere contralateral to the stenosis/occlusion in all NIRS contrasts. In addition, the number of connections in the contralateral side was 149 $\pm 9 \%$ (mean $\pm \mathrm{SD}$ ) higher than in the hemisphere ipsilateral to the stenosis/occlusion. Together, these findings suggest that in order to keep the brain's global stability and overcome the lower flow due to the stenosis/occlusion, the brain might have to reorganize itself by increasing the connectivity and information transfer in the contralateral hemisphere. Although we believe that more patients are needed to better understand the common aspects of atherosclerotic disease in the brain, this work 
progresses towards a novel method that can provide useful information for assessing cerebral hemodynamics in patients diagnosed with atherosclerotic disease, both during functional tasks and in the resting state.

\section{Conclusions}

In this work we employed nearinfrared spectroscopy (NIRS) to further comprehend how atherosclerotic disease affects brain hemodynamics, both at rest and during breath holding. We showed that patients diagnosed with unilateral stenosis have impaired hemodynamic response, presenting lower vasoreactivity and lack of coherent spontaneous fluctuations in the hemisphere ipsilateral to the stenosis. Patients with a carotid occlusion present more heterogeneous responses, possibly related to the increased collateral circulation in the hemisphere ipsilateral to the occlusion. Unlike healthy subjects, NIRS functional connectivity analysis during the resting state suggests that patients with atherosclerotic disease have interconnected regions of spontaneous hemodynamic fluctuations that can be very heterogeneous. Overall, our results suggest that NIRS can open new directions to the investigation of the effects of atherosclerotic disease in the patient's brain, and larger scale clinical trials should further substantiate our findings.

\section{Acknowledgments}

This work was primarily supported by the São Paulo Research Foundation (FAPESP) through 2012/02500-8 (RCM) and 2013/07559-3 (RJMC, FC, RCM). Additional support was obtained from the Coordination for the Improvement of Higher Education Personnel (CAPES). 
Medical Research Archives, Vol. 5, Issue 6, June 2017

Use of near-infrared spectroscopy to probe occlusion severity in patients diagnosed with carotid atherosclerotic disease

\section{References}

Babikian, V.L., Hyde, C., Pochay, V., Winter, M.R., 1994. Clinical correlates of high-intensity transient signals detected on transcranial Doppler sonography in patients with cerebrovascular disease. Stroke 25, 1570 LP-1573.

Barrat, A., Barthélemy, M., Pastor-Satorras, R., Vespignani, A., 2004. The architecture of complex weighted networks. Proc. Natl. Acad. Sci. U. S. A. 101, 3747-3752. doi:10.1073/pnas.0400087101

Brigadoi, S., Ceccherini, L., Cutini, S., Scarpa, F., Scatturin, P., Selb, J., Gagnon, L., Boas, D.A., Cooper, R.J., 2014. Motion artifacts in functional near-infrared spectroscopy: A comparison of motion correction techniques applied to real cognitive data. Neuroimage 85, 181-191. doi:10.1016/j.neuroimage.2013.04.082

Carbonell, F., Bellec, P., Shmuel, A., 2011. Global and system-specific resting-state fMRI fluctuations are uncorrelated: principal component analysis reveals anti-correlated networks. Brain Connect. 1, 496-510. doi:10.1089/brain.2011.0065

Carr, S., Farb, A., Pearce, W.H., Virmani, R., Yao, J.S.T., 1996. Atherosclerotic plaque rupture in symptomatic carotid artery stenosis. J. Vasc. Surg. 23, 755766.

doi:http://dx.doi.org/10.1016/S07415214(96)70237-9

Carroll, B.A., 1991. Carotid sonography. Radiology 178, 303-313.

de Weerd, M., Greving, J.P., Hedblad, B., Lorenz, M.W., Mathiesen, E.B., O\&\#039;Leary, D.H., Rosvall, M.,
Sitzer, M., Buskens, E., Bots, M.L., 2010. Prevalence of Asymptomatic Carotid Artery Stenosis in the General Population. Stroke 41, 1294 LP-1297.

Debrey, S.M., Yu, H., Lynch, J.K., Lövblad, K.-O., Wright, V.L., Janket, S.-J.D., Baird, A.E., 2008. Diagnostic Accuracy of Magnetic Resonance Angiography for Internal Carotid Artery Disease. Stroke 39, 2237 LP-2248.

Delcker, A., Diener, H.C., Wilhelm, H., 1997. Source of cerebral microembolic signals in occlusion of the internal carotid artery. J. Neurol. 244, 312-317. doi:10.1007/s004150050093

Demchuk, a M., Christou, I., Wein, T.H., Felberg, R. a, Malkoff, M., Grotta, J.C., Alexandrov, a V, 2000. Specific transcranial Doppler flow findings related to the presence and site of arterial occlusion. Stroke. 31, 140-146. doi:10.1161/01.STR.31.1.140

Duncan, G.W., Pessin, M.S., Mohr, J.P., Adams, R.D., 1976. Transient cerebral ischemic attacks. Adv. Intern. Med. 21, $1-20$.

Favilla, C.G., Mesquita, R.C., Mullen, M., Durduran, T., Lu, X., Kim, M.N., Minkoff, D.L., Kasner, S.E., Greenberg, J.H., Yodh, A.G., Detre, J.A., 2014. Optical bedside monitoring of cerebral blood flow in acute ischemic stroke patients during headof-bed manipulation. Stroke 45, 12691274.

doi:10.1161/STROKEAHA.113.00411

6

Ferrari, M., Quaresima, V., 2012. A brief review on the history of human functional near-infrared spectroscopy (fNIRS) development and fields of 
application. Neuroimage 63, 921-935. doi:10.1016/j.neuroimage.2012.03.049

Fisher, C.M., 1962. Concerning Recurrent Transient Cerebral Ischemic Attacks. Can. Med. Assoc. J. 86, 1091-1099.

Franceschini, M.A., Boas, D.A., 2004. Noninvasive measurement of neuronal activity with near-infrared optical imaging. Neuroimage 21, 372-386. doi:http://dx.doi.org/10.1016/j.neuroim age.2003.09.040

Gagnon, L., Cooper, R.J., Yücel, M.A., Perdue, K.L., Greve, D.N., Boas, D.A., 2012. Short separation channel location impacts the performance of short channel regression in NIRS. Neuroimage 59, 2518-2528. doi:10.1016/j.neuroimage.2011.08.095

Gagnon, L., Yücel, M.A., Boas, D.A., Cooper, R.J., 2014. Further improvement in reducing superficial contamination in NIRS using double short separation measurements. Neuroimage $\quad 85, \quad 127-135$. doi:10.1016/j.neuroimage.2013.01.073

Garrity, A.G., Pearlson, G.D., McKiernan, K., Lloyd, D., Kiehl, K.A., Calhoun, V.D., 2007. Aberrant "default mode" functional connectivity in schizophrenia. Am. J. Psychiatry 164, 450-457.

doi:10.1176/appi.ajp.164.3.450

Greicius, M.D., Supekar, K., Menon, V., Dougherty, R.F., 2009. Resting-state functional connectivity reflects structural connectivity in the default mode network. Cereb. Cortex 19, 7278. doi:10.1093/cercor/bhn059

Hankey, G.J., Warlow, C.P., Sellar, R.J., 1990. Cerebral angiographic risk in mild cerebrovascular disease. Stroke 21, 209 LP-222.
Hollander, M., Bots, M.L., Del Sol, A.I., Koudstaal, P.J., Witteman, J.C.M., Grobbee, D.E., Hofman, A., Breteler, M.M.B., 2002. Carotid plaques increase the risk of stroke and subtypes of cerebral infarction in asymptomatic elderly. Circulation 105, 2872-2877.

Huppert, T.J., Diamond, S.G., Franceschini, M.A., Boas, D.A., 2009. HomER: a review of time-series analysis methods for near-infrared spectroscopy of the brain. Appl. Opt. 48, 280-298. doi:10.1016/j.drugalcdep.2008.02.002. A

Jobsis, F.F., 1977. Noninvasive, infrared monitoring of cerebral and myocardial oxygen sufficiency and circulatory parameters. Science (80-. ). 198, 1264 LP-1267.

Larsen, F.S., Olsen, K.S., Hansen, B. a, Paulson, O.B., Knudsen, G.M., 1994. Transcranial Doppler is valid for determination of the lower limit of cerebral blood flow autoregulation. Stroke 25, 1985-8. doi:10.1161/01.STR.25.10.1985

Li, Z., Liu, H., Liao, X., Xu, J., Liu, W., Tian, F., He, Y., Niu, H., 2015. Dynamic functional connectivity revealed by resting-state functional near-infrared spectroscopy 6, 1575715767. doi:10.1364/BOE.6.002337

Lu, C.M., Zhang, Y.J., Biswal, B.B., Zang, Y.F., Peng, D.L., Zhu, C.Z., 2010. Use of fNIRS to assess resting state functional connectivity. J. Neurosci. Methods 186, 242-249. doi:10.1016/j.jneumeth.2009.11.010

Markus, H.S., Harrison, M.J., 1992. Estimation of cerebrovascular reactivity using transcranial Doppler, including the use of breath-holding as the vasodilatory stimulus. Stroke 23, 668 
Medical Research Archives, Vol. 5, Issue 6, June 2017

Use of near-infrared spectroscopy to probe occlusion severity in patients diagnosed with carotid atherosclerotic disease

LP-673.

Mesquita, R.C., Franceschini, M.A., Boas, D.A., 2010. Resting state functional connectivity of the whole head with near infrared spectroscopy. Biomed. Opt. $\quad$ Express 324-336. doi:10.1364/BOE.1.000324

Mesquita, R.C., Han, S.W., Miller, J., Schenkel, S.S., Pole, A., Esipova, T. V., Vinogradov, S.A., Putt, M.E., Yodh, A.G., Busch, T.M., 2012. Tumor blood flow differs between mouse strains: Consequences for vasoresponse to photodynamic therapy. PLoS One 7, 110. doi:10.1371/journal.pone.0037322

Mesquita, R.C., Huppert, T.J., Boas, D. a, 2009. Exploring neuro-vascular and neuro-metabolic coupling in rat somatosensory cortex. Phys. Med. Biol. 54, 175-85. doi:10.1088/0031$9155 / 54 / 2 / 001$

Mesquita, R.C., Putt, M., Chandra, M., Yu, G., Xing, X., Han, S.W., Lech, G., Shang, Y., Durduran, T., Zhou, C., Yodh, A.G., Mohler, E.R., 2013. Diffuse optical characterization of an exercising patient group with peripheral artery disease. J. Biomed. Opt. 18, 57007. doi:10.1117/1.JBO.18.5.057007

Mesquita, R.C., Yodh, A.G., 2011. Diffuse Optics: Fundamentals \& Tissue Applications. Proc. Int. Sch. Phys. "Enrico Fermi", R. Kaiser, D.S. Wiersma, L. Fallani, eds.IOS, Amsterdam. doi:10.3254/978-1-60750755-0-51

Minati, L., Kress, I.U., Visani, E., Medford, N., Critchley, H.D., 2011. Intra- and extra-cranial effects of transient blood pressure changes on brain near-infrared spectroscopy (NIRS) measurements. J. Neurosci. Methods 197, 283-288. doi:10.1016/j.jneumeth.2011.02.029
Müller, M., Schimrigk, K., 1996. Vasomotor Reactivity and Pattern of Collateral Blood Flow in Severe Occlusive Carotid Artery Disease. Stroke 27, 296 LP-299.

Newman, M.E.J., 2004. Analysis of weighted networks. Phys. Rev. E - Stat. Nonlinear, Soft Matter Phys. 70. doi:10.1103/PhysRevE.70.056131

Norrving, B., Nilsson, B., Risberg, J., 2015. rCBF in patients with carotid occlusion. Resting and hypercapnic flow related to collateral pattern. Stroke. 13, 155-162. doi:10.1161/01.STR.13.2.155

North American Symptomatic Carotid Endarterectomy Trial(NASCET) Steering Committee, 1991. Original Contributions North American Symptomatic Carotid Endarterectomy Trial.

Novi, S.L., Rodrigues, R.B.M.L., Mesquita, R.C., 2016. Resting state connectivity patterns with near- infrared spectroscopy data of the whole head. Biomed Opt Express 7, 2524-2537. doi:10.1364/BOE.7.002524

O’Leary, D.H., Polak, J.F., Kronmal, R.A., Kittner, S.J., Bond, M.G., Wolfson, S.K., Bommer, W., Price, T.R., Gardin, J.M., Savage, P.J., 1992. Distribution and correlates of sonographically detected carotid artery disease in the Cardiovascular Health Study. The CHS Collaborative Research Group. Stroke 23, 1752 LP-1760.

Obrig, H., Neufang, M., Wenzel, R., Kohl, M., Steinbrink, J., Einhäupl, K., Villringer, A., 2000. Spontaneous Low Frequency Oscillations of Cerebral Hemodynamics and Metabolism in Human Adults. Neuroimage 12, 623639. doi:10.1006/nimg.2000.0657

Oscillators, C., 2010. Europe PMC Funders 
Medical Research Archives, Vol. 5, Issue 6, June 2017

Use of near-infrared spectroscopy to probe occlusion severity in patients diagnosed with carotid atherosclerotic disease

Group Coupled Oscillators : 26, 25-29.

Philip, K.J., H., R.A., C., H.R., 1984.

Therapy of Ischemic Cerebral Vascular

Disease Due to Atherothrombosis. N.

Engl. J. Med. 311, 100-105. doi:10.1056/NEJM198407123110206

Ratnatunga, C., Adiseshiah, M., 1990. Increase in middle cerebral artery velocity on breath holding: A simplified test of cerebral perfusion reserve. Eur. J. Vasc. Surg. 4, 519-523. doi:10.1016/S0950-821X(05)80795-9

Ringelstein, E.B., Sievers, C., Ecker, S., Schneider, P.A., Otis, S.M., 1988. Noninvasive assessment of $\mathrm{CO} 2-$ induced cerebral vasomotor response in normal individuals and patients with internal carotid artery occlusions. Stroke 19, 963 LP-969.

Rothwell, P.M., Gibson, R.J., Slattery, J., Sellar, R.J., Warlow, C.P., 1994. Equivalence of measurements of carotid stenosis. A comparison of three methods on 1001 angiograms. European Carotid Surgery Trialists' Collaborative Group. Stroke 25, 2435 LP-2439.

Sabeti, S., Schillinger, M., Mlekusch, W., Willfort, A., Haumer, M., Nachtmann, T., Müllner, M., Lang, W., Ahmadi, R., Minar, E., 2004. Quantification of Internal Carotid Artery Stenosis with Duplex US: Comparative Analysis of Different Flow Velocity Criteria. Radiology 232, 431-439. doi:10.1148/radiol.2321030791

Sakakibara, E., Homae, F., Kawasaki, S., Nishimura, Y., Takizawa, R., Koike, S., Kinoshita, A., Sakurada, H., Yamagishi, M., Nishimura, F., Yoshikawa, A., Inai, A., Nishioka, M., Eriguchi, Y., Matsuoka, J., Satomura, Y., Okada, N., Kakiuchi, C., Araki, T.,
Kan, C., Umeda, M., Shimazu, A., Uga, M., Dan, I., Hashimoto, H., Kawakami, N., Kasai, K., 2016. Detection of resting state functional connectivity using partial correlation analysis: A study using multi-distance and wholehead probe near-infrared spectroscopy. Neuroimage 142, 590-601. doi:10.1016/j.neuroimage.2016.08.011

Sanborn, M.R., Edsell, M.E., Kim, M.N., Mesquita, R., Putt, M.E., Imray, C., Yow, H., Wilson, M.H., Yodh, A.G., Grocott, M., Martin, D.S., 2015. Cerebral Hemodynamics at Altitude: Effects of Hyperventilation and Acclimatization on Cerebral Blood Flow and Oxygenation. Wilderness Environ. Med. 26, 133-141. doi:10.1016/j.wem.2014.10.001

Sasai, S., Homae, F., Watanabe, H., Sasaki, A.T., Tanabe, H.C., Sadato, N., Taga, G., 2012. A NIRS-fMRI study of resting state network. Neuroimage 63, 179-193.

doi:10.1016/j.neuroimage.2012.06.011

Scholkmann, F., Kleiser, S., Metz, A.J., Zimmermann, R., Mata Pavia, J., Wolf, U., Wolf, M., 2014. A review on continuous wave functional nearinfrared spectroscopy and imaging instrumentation and methodology. Neuroimage 85, 6-27. doi:10.1016/j.neuroimage.2013.05.004

Silvestrini, M., Paolino, I., Vernieri, F., Pedone, C., Baruffaldi, R., Gobbi, B., Cagnetti, C., Provinciali, L., Bartolini, M., 2009. Cerebral hemodynamics and cognitive performance in patients with asymptomatic carotid stenosis. Neurology 72, 1062-1068. doi:10.1212/01.wnl.0000345015.35520. 52

Silvestrini, M., Troisi, E., Matteis, M., Cupini, L.M., Caltagirone, C., 1996. 
Medical Research Archives, Vol. 5, Issue 6, June 2017

Use of near-infrared spectroscopy to probe occlusion severity in patients diagnosed with carotid atherosclerotic disease

Transcranial Doppler Assessment of Cerebrovascular Reactivity in Symptomatic and Asymptomatic Severe Carotid Stenosis. Stroke 27, 1970 LP-1973.

Soinne, L., Helenius, J., Tatlisumak, T., Saimanen, E., Salonen, O., Lindsberg, P.J., Kaste, M., 2003. Cerebral Hemodynamics in Asymptomatic and Symptomatic Patients With High-Grade Carotid Stenosis Undergoing Carotid Endarterectomy. Stroke 34, 1655 LP1661.

Stam, C.J., Jones, B.F., Nolte, G., Breakspear, M., Scheltens, P., 2007. Small-world networks and functional connectivity in Alzheimer's disease. Cereb. Cortex 17, 92-99. doi:10.1093/cercor/bhj127

Störk, S., van den Beld, A.W., von Schacky, C., Angermann, C.E., Lamberts, S.W.J., Grobbee, D.E., Bots, M.L., 2004. Carotid Artery Plaque Burden, Stiffness, and Mortality Risk in Elderly Men. Circulation 110, 344 LP-348.

Tachtsidis, I., Leung, T.S., Devoto, L., Delpy, D.T., Elwell, C.E., 2008. Measurement of Frontal Lobe Functional Activation and Related Systemic Effects: ANearInfraredSpectroscopy Investigation. Adv. Exp. Med. Biol. 614, 397-403. doi:10.1007/978-0-387-74911-2

Tian, L., Meng, C., Jiang, Y., Tang, Q., Wang, S., Xie, X., Fu, X., Jin, C., Zhang, F., Wang, J., 2016. Abnormal functional connectivity of brain network hubs associated with symptom severity in treatment-naive patients with obsessive-compulsive disorder: A resting-state functional MRI study. Prog. Neuro-Psychopharmacology Biol. Psychiatry 66, 104-111. doi:10.1016/j.pnpbp.2015.12.003
Trial, M.R.S.E.C.S., 1991. interim results for symptomatic patients with severe (70$99 \%)$ or with mild (0-29\%) carotid stenosis: Europen Carotid Surgery Trialists Collaborative Group. Lancet 337, 1235-1243.

Tsivgoulis, G., Sharma, V.K., Lao, A.Y., Malkoff, M.D., Alexandrov, A. V., 2007. Validation of transcranial Doppler with computed tomography angiography in acute cerebral ischemia. Stroke 38, 1245-1249. doi:10.1161/01.STR.0000259712.6477 2.85

Vasdekis, S.N., Tsivgoulis, G., Athanasiadis, D., Andrikopoulou, A., Voumvourakis, K., Lazaris, A.M., Stamboulis, E., 2012. Cerebrovascular reacivity assessment in patients with carotid artery disease: A combined TCD and NIRS study. J. Neuroimaging 22, 261265.

6569.2011.00595.x

doi:10.1111/j.1552-

Vernieri, F., Pasqualetti, P., Passarelli, F., Rossini, P.M., Silvestrini, M., 1999. Outcome of Carotid Artery Occlusion Is Predicted by Cerebrovascular Reactivity. Stroke 30, 593 LP-598.

Wardlaw, J.M., 2006. NHS Research and Development Health Technology Assessment Carotid Stenosis Imaging Group: Non-invasive imaging compared with intra-arteril angiography in the diagnosis of symptomatic carotid stenosis: A meta-analysis. Lancet 367, 1503-1512. doi:10.1016/S01406736(06)68650-9

Widder, B., Kleiser, B., Krapf, H., 1994. Course of cerebrovascular reactivity in patients with carotid artery occlusions. Stroke 25, 1963 LP-1967.

Wilterdink, J.L., Feldmann, E., Furie, K.L., Bragoni, M., Benavides, J.G., 1997. 
Medical Research Archives, Vol. 5, Issue 6, June 2017

Use of near-infrared spectroscopy to probe occlusion severity in patients diagnosed with carotid atherosclerotic disease

Transcranial Doppler Ultrasound Battery Reliably Identifies Severe Internal Carotid Artery Stenosis. Stroke 28, 133 LP-136.

Yodh, A.G., Boas, D. a, 2003. Functional imaging with diffusing light. Biomed. Photonics Handb. 1-45.

Zhang, Y., Wang, L., Yang, J., Yan, R., Zhang, J., Sang, L., Li, P., Liu, H., Qiu, M., 2017. Abnormal Functional Networks in Resting-State of the Subcortical Chronic Stroke Patients with Hemiplegia. Brain Res. doi:10.1016/j.brainres.2017.02.012
Zhu, G., Yuan, Q., Yang, J., Yeo, J.H., 2015. The role of the circle of Willis in internal carotid artery stenosis and anatomical variations: a computational study based on a patient-specific threedimensional model. Biomed. Eng. Online 14, 107. doi:10.1186/s12938015-0105-6

Zwiebel, W.J., 1992. Duplex sonography of the cerebral arteries: efficacy, limitations, and indications. Am. J. Roentgenol. 158, 29-36. doi:10.2214/ajr.158.1.1727355 